Article

\title{
DEM Investigation of the Influence of Particulate Properties and Operating Conditions on the Mixing Process in Rotary Drums: Part 2-Process Validation and Experimental Study
}

\author{
Jakub Hlosta *(1), Lucie Jezerská@, Jiř́i Rozbroj, David Žurovec, Jan Nečas and Jiří Zegzulka \\ ENET Centre, Bulk Solids Centre-Czech Republic, VSB-Technical University of Ostrava, 17. listopadu 15/2172, \\ 70833 Ostrava, Czech Republic; lucie.jezerska@vsb.cz (L.J.); jiri.rozbroj@vsb.cz (J.R.); \\ david.zurovec@vsb.cz (D.Ž.); jan.necas@vsb.cz (J.N.); jiri.zegzulka@vsb.cz (J.Z.) \\ * Correspondence: jakub.hlosta@vsb.cz; Tel.: +420-597-329-371
}

Received: 7 January 2020; Accepted: 26 January 2020; Published: 5 February 2020

check for updates

\begin{abstract}
The process of homogenization of particulates is an indispensable part of many industrial processes, and, therefore, it is necessary to pay a special attention to this area and develop it. This paper deals with a complex study of homogenization of particulate matters in a rotary drum in terms of shape, size, and density of particles. In addition, the influence of operating parameters, such as drum filling capacity, rotational speed, and drum filling pattern are also investigated. Studies of reproducibility of discrete element method simulations, effects of rotary drum sizes or effects of drum volumetric filling to the mixture homogeneity index were also carried out. In general, the least satisfactory values of the homogeneity index resulted from the mixing of particles with different densities. The dominating factor of homogenization was the drum filling-up degree. The course of the homogeneity index in 140,280, and $420 \mathrm{~mm}$ drums was very similar and after five revolutions of the drum, identical values of the homogeneity index were achieved for all the drum diameters. The optimal drum filling-up degree is at $40-50 \%$ for the spherical particles and $30-40 \%$ for the sharp-edged particles. The repeatability of simulations showed the maximum relative standard deviation of the homogeneity index at $0.6 \%$ from ten simulation repetitions with the same parametric conditions.
\end{abstract}

Keywords: DEM; homogenization; mixing; rotating drum; particulates; validation

\section{Introduction}

In various industries, starting from pharmaceutical companies, processing of raw materials, agriculture, food processing and ending with civil engineering and many other branches, the product quality often depends on the quality of mixing of particulate substances. For many reasons, the homogeneous mixing of particles represents a key factor for a wide range of operations, such as intensification of chemical reactions, improvements in mechanical properties (e.g., spatial distribution of particle in concrete materials), API (active pharmaceutical ingredient) uniform distribution in dosage forms, uniform distribution of color pigments in paints and beauty products, constant flavor in the whole volume of flavoring preparations, etc. Therefore, the key prerequisite for the successful mixing of particulate substances is homogeneity of the resulting products achieved by online monitoring. To determine the resulting mixture quality, in general, the homogeneity index was introduced $[1,2]$. The homogeneity index correct value should be closely interconnected with specific properties of resulting mixtures regardless of the mixing process type used, and, in addition, its easy and precise determination should be provided. However, more than 40 various methods have been proposed for the homogeneity index determination by various authors so far. The number of factors influencing the 
degree of mixing points out to the complexity of the mixing process and difficulties in the effort of obtaining the optimal homogeneity and resulting mixture properties [3].

Among other things, the rotary drum is quite often used for mixing of particulate materials [4-6]. Depending on the mixer volumetric filling, drum rotational speed and friction or cohesive forces of the material [7], movements of particulate materials in this mixer can be characterized by several ways. In the case of a very low degree of volumetric filling, and low drum speed, the material will only slide along the drum wall (sliding or surging motion). With increasing drum speed, friction forces augmented by the centrifugal force will prevail and the material will rise and descend along the drum wall repeatedly (slumping). In addition, the dynamic angle of repose of the material will be formed and the material will be rolling. As the volumetric filling and revolution increase, a transition to the cascade and gravity takes place and the material fall over in waves back to the drum bottom section [7]. The rolling, cascading, and cataracting movement modes are most suitable for industrial mixing processes (see Figure 1) [8,9].

\begin{tabular}{|c|c|c|c|c|c|c|c|}
\hline Basic Form & \multicolumn{2}{|c|}{ Slippping motion } & \multicolumn{3}{c|}{ Cascading motion } & \multicolumn{2}{c|}{ Cataracting motion } \\
\hline Subtype & Sliding & Surging & Slumping & Rolling & Cascading & Cataracting & Centrifuging \\
\hline & & & &
\end{tabular}

Figure 1. Types of material movements in the rotary drum [7].

In the rolling mode, particle bed can be divided into two zones, namely the active zone and the static zone [8]. Since the mixing behavior mainly occurs in the active region, particle motions in this region are crucial to evaluate the mixing performance of the whole drum. Depending on the operating drum revolutions, various movement types were defined for freely flowing particulate materials in the rotary drum. Among others, the criterion for individual types of movement is also the ratio of centrifugal and gravity forces affecting the particles, i.e., the Froude number [7].

The studies of mixing of materials deal with various parameters influencing the kinetics and equilibria of this process. For instance, by examining of the active region thickness based on comparisons of the theoretic model with experimental results of velocity fields of particles in the rotary drum $[8,10]$. Further studies point out that the quality and performance of mixing of particles in the drum are related to some variables, such as particle sizes and densities, friction coefficient, degree of drum filling, and rotary speed [11-14]. The discrete element method (DEM) simulations are used for mixing processes [14-18] because they are able to provide more heterogeneous and detailed data than the experimental results. At present, the ever-growing capacity of computers enables precise, universal and continuous simulation and monitoring of the mixing process. Therefore, the DEM methods are widely used for this purpose and its significantly increased application in the recent years is evidenced by the increasing number of publications.

The relevance between the active layer of mixing and the total mixing in the rotary drum, or mixing modes, was also studied by the use of DEM [18-20]. There is an effort for high quality graphic evaluations of the particle arrangements depending on various process variables starting with the revolutions and ending with the drum filling degree. The course of the mixing process during such experiment is usually observed in 2D only. In order to evaluate the volume homogeneity, it is then necessary to choose a suitable sampling method, which is not often an easy task. In order to avoid "dead zones," the use of passive elements placed inside the rotary drum is offered for practical applications $[17,21]$. Using the DEM, the mixing process can be analyzed in detail without the need to 
design intricately a prototype and substantial experimental works. Studies dealing with the energy consumption during mixing processes in the rotary drums based on the DEM are also common $[15,22]$ because it is an important design parameter for the motors of drum mixing systems. In most cases, it is taken for granted that the particles are of the same size and/or uniformly distributed and that no axial tilting for the rotary drum is needed. In practice, such assumptions may be true, or maybe not. In order to study the energy demand of a polydisperse mixture of various degrees of volumetric filling of the drum, the DEM model can be used. Using the numerical model based on the DEM method, the particle segregation in the rotary drum can be also investigated. Segregation mechanisms in mixing of cohesive and non-cohesive particles are also analyzed in this way [16,23]. In addition, models for the description of mixing processes the diffusion equation [24]. However, there is still only a few of papers dealing with experimentally verified DEM studies of a greater complexity, where a larger number of parameters is involved. Therefore, this paper is focused in this direction, which fills the research gap in the description of particle mixing in the rotary drum for combinations of several operating parameters at the same time.

This paper presents a study of the influence of particle size, shape, density, method, and degree of filling, and rotational speed of the rotary drum to the process of mixing. The mixing quality is quantitatively evaluated by the homogeneity index. The rotary drum was selected as a representative of the diffusion mixing mechanism. It is an example relatively easily applicable to the possibilities of using the DEM method in both industry and research. The first part of the study was focused on calibration and validation of the DEM numerical model. In the second part, all influences of the above parameters of the homogeneity index are discussed in detail. Optimum values and conditions were evaluated and discussed, as well as the possibilities in combinations of particle shapes, sizes, and densities, based-for instance-on illustrations of sections from the inside of the rotating drum, as a possibility to acquire data of particle assemblies at an arbitrary moment of mixing.

\section{Materials and Methods}

Seven various particulate materials were used in the study. The selection of materials represented particles of different sizes, shapes, and densities. These particulate materials are shown in Figure 2. The selection includes various geometrical bodies, such as spheres, cubes, or cylinders that reflect the morphology of real particles in the industrial practice. In the spherical shape, a range of agricultural products can be shown - pea, chickpea, nuts, various seeds, etc. The cylindrical shape represents the wooden pellets in the energy industry (biomass processing), and the cubic shapes then sharp-edged particles of crushed stone, ore, or coal.

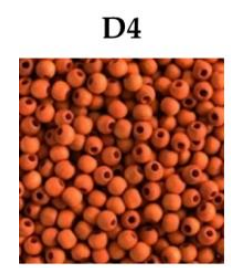

wood - ash spherical

$4 \mathrm{~mm}$

$0.02 \mathrm{~g}$
D6

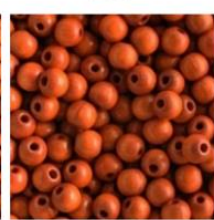

wood - ash spherical $6 \mathrm{~mm}$ $0.07 \mathrm{~g}$
D12

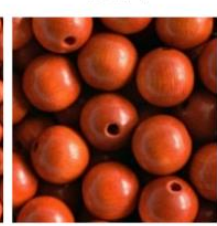

wood - ash spherical $12 \mathrm{~mm}$ $0.69 \mathrm{~g}$

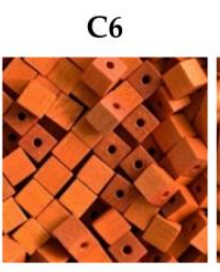

wood - ash cubical $6 \times 6 \times 6 \mathrm{~mm}$ $0.14 \mathrm{~g}$
P6x15

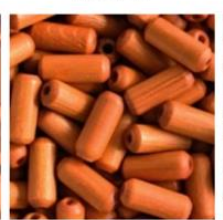

wood - ash cylindrical $6 \times 15 \mathrm{~mm}$ $0.28 \mathrm{~g}$

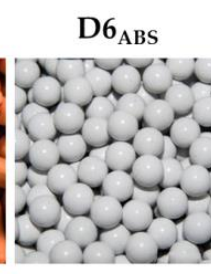

ABS

spherical

$6 \mathrm{~mm}$

$0.20 \mathrm{~g}$
D5 $5_{\text {steel }}$

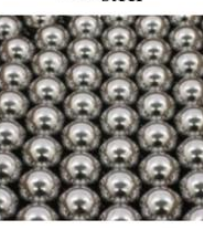

steel

spherical

$5 \mathrm{~mm}$

$0.50 \mathrm{~g}$

Figure 2. Particulate materials and their basic properties.

The experiments were conducted in the rotary drum (Figure 3a). The assembly consists of a frame made of aluminum beams, on which an electric motor with a gearbox driving the drum is mounted. The drum is freely laid on four wheels placed on the frame. The driving torque is transferred by a key and keyseat inside the shaft. The electric motor is connected to the mains over a frequency converter, which enables the revolution control. The drum inner diameter of $140 \mathrm{~mm}$ was chosen with regard to 
the size of the used particulate materials. Approximately 25 particles of $6 \mathrm{~mm}$ size each were added corresponding to $140 \mathrm{~mm}$ diameter crosswise the surface profile. Adequate number of particles is important for the determination of the dynamic angle of repose. On the other hand, if there were too many particles in the drum, the DEM calculation times would increase significantly [25]. Inside the drum, there is a partition dividing the drum into two parts. The shorter part is $30 \mathrm{~mm}$ wide and it serves mainly for calibration experiments. This size approximately corresponds to five particles (of $6 \mathrm{~mm}$ size), which is sufficient for the calibration experiments and still helps to avoid the undesirable increase in the calculation times. The second part is $215 \mathrm{~mm}$ wide and it serves for studying of mixing processes in rotary drum mixers, where it is possible to monitor the material distribution also in the axial direction. The drum rotary speed can be controlled by the frequency converter, where the range of drum revolutions is from 6 to $36 \mathrm{rpm}$. The gearbox is advantageously used for the increased driving torque due to which the rotary speed is not influenced by the weight of particulate materials. The whole drum is made of transparent and white PMMA (poly-methyl methacrylate = plexiglass) so that it is possible to analyze mechanisms and mixing zones (Figure 3b). Transparent as well as white PMMA parts show the same frictional properties.

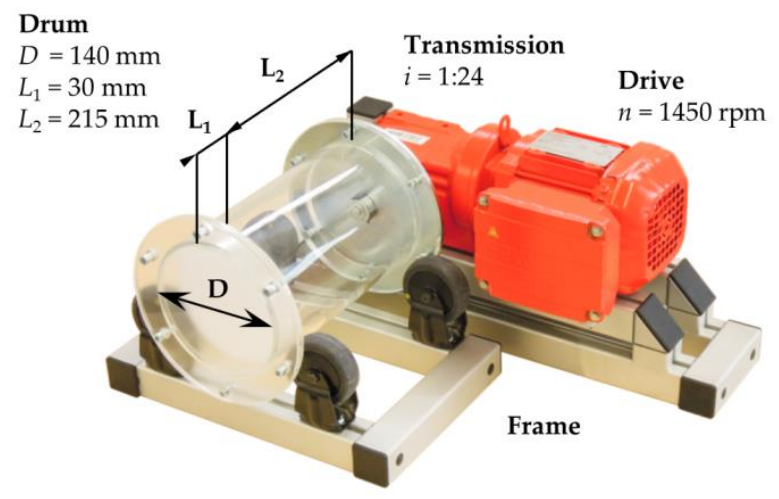

(a)

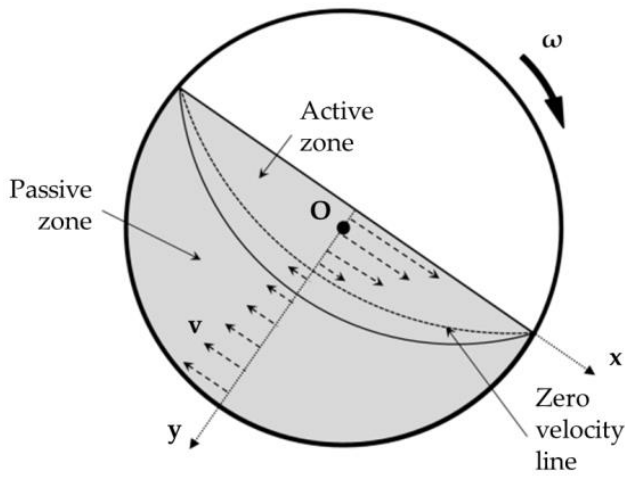

(b)

Figure 3. Rotary drum: (a) experimental rotary drum assembly; (b) schematic representation of active and passive mixing zones.

The analyses of material movements inside the drum during the experiments were conducted with the use of a high-speed camera and the PIV (particle image velocimetry) analysis. A typical way of the material rolling movements in the rotary drum is shown in Figure $3 \mathrm{~b}$. The PIV method is an optical method based on flow visualization. If sufficiently small particles are used, where it is assumed that they truly copy the flow dynamics, the method is utilized for measurements of instantaneous velocities of flowing of fluids and gases, but it can be also used for visualization of bulk material. The monitored medium is lighted in a way making the drifting particles as visible as possible, and consequently, the movement of particles is used for the conversion of the speed and direction (velocity field) of the flow. The measurement results yielded by the PIV method represent either two-dimensional or also three-dimensional vector fields. At least two frame exposures are necessary for the flow analysis. Then it will be possible to calculate the shift vector per each region created in this way using the autocorrelation or cross-correlation methods. In this procedure, the velocity will be determined by the use of the time elapsed between the frames and the physical size of each pixel of the captured image. This method brings about a major advantage by the fact that the measuring equipment itself does not influence the examined flowing streams. On the other hand, the method disadvantage is that it is not possible to measure the particle velocity in the direction from/to the camera. Particle movements in this direction cannot be eliminated in any other way and it may therefore cause a distortion within the measured plane. This problem is eliminated with the stereoscopic PIV, where within the use of two cameras all three components of the movement are monitored [26]. 


\subsection{Tested Parameters}

This study compares the mixing process course for selected couples of particulate materials and the influence of filling patterns, drum revolutions, shape, size, and density of particles to the resulting homogeneity index of the mixture [27]. Seven couples of particulate materials were selected from the collection. These couples were examined in the experimental studies. The separate couples were selected for their respective shapes, sizes, and densities. Three degrees of volumetric filling of the drum with the material were tested at 25,50 , and $75 \%$. In addition, three drum revolving speeds represent three modes of movement of the material in the drum. Sliding, rolling, and cascade movements were represented by the drum revolutions at $6,21.6$, and $36 \mathrm{rpm}$. The last monitored attribute was either the vertical or the horizontal method of filling the mixing drum.

\subsection{Evaluation of Homogeneity}

Validation experiments for all seven couples of particulate materials were conducted with $50 \%$ volumetric filling of the drum, at 6 and $21.6 \mathrm{rpm}$ and both vertical and horizontal filling. For all 126 parameter combinations simulation models were created, out of which the resulting data were obtained. As a quantifiable parameter determining the degree of mixing of two groups of particles, the homogeneity index was chosen. This index is sometimes called the segregation (mixing) index defined by the number of contacts among the particles:

$$
S=\frac{C_{A A}}{C_{A A}+C_{A B}}+\frac{C_{B B}}{C_{B B}+C_{A B}}
$$

where $C_{\mathrm{AA}}$ is the number of contacts among the particles $\mathrm{A}, C_{\mathrm{BB}}$ the number of contacts among the particles $\mathrm{B}$, and $C_{\mathrm{AB}}$ is number of contacts among the particles $\mathrm{A}$ and $\mathrm{B}$. The homogeneity index then assumes the value within the range $0<S<2$, where at random mixtures the value fluctuates around $S$ $\approx 1$. The values lying on both sides from 1 are transient segregation states $(S>1)$ and at an ordered mixed system $(S<1)$. The value $S=0$ corresponds to an ideally ordered system, where not $C_{\mathrm{AA}}$ or $C_{\mathrm{BB}}$ contacts exist [28-30].

Each of the validation experiments and simulation models were carried out for five drum revolutions, i.e., for rotation of $5 \times 360^{\circ}$. After the five drum revolutions, the resulting homogeneity index was determined. At 28 validation experiments the drum was always filled up to its $25 \%$ of the total volume capacity $(460 \mathrm{~mL}$ ) with the A particles and up to its $25 \%$ of the total volume capacity with the B particles. After this, the whole charge was mixed by five revolutions of the drum. The whole experiment was recorded by a camera at the frequency of 120 frames per second. For the validation experiment evaluations and comparisons with numerical models, 11 frames were taken out of the record in times from $t_{0}$ to $t_{10}$ from the drum start-up always after $\frac{1}{2}$ drum revolution. As the mutual movement of particles in the drum is strongly stochastic phenomenon, the correctness of the simulations was evaluated individually based on the particle movement behavior in the drum and graphical comparisons of the mixing quality in separate movement phases from $t_{0}$ to $t_{10}$.

\subsection{Numerical Modelling}

The DEM model used in this study uses the so-called "soft-sphere" method originally developed by Cundall and Strack. In this method, the particles in contact are capable of resisting to small deformations and these deformations are used for calculations of forces having effects among the particles. Tsuji et al. (1992) proposed a non-linear contact model by adaptation of the original model proposed by Cundall and Strack [31]. The currently plentifully used model of Hertz-Mindlin is in commercial software EDEM characterized by the following Equations (2) and (3). This model is suitable for simulations of coarse-grained and non-cohesive materials.

$$
F_{i j}^{n}=-\frac{4}{3} E^{*} \sqrt{R^{*}} \delta_{i j}^{n \frac{3}{2}}-2 \sqrt{\frac{5}{6}} \psi \sqrt{k_{n} m^{*}} v_{i j}^{n}
$$




$$
F_{i j}^{t}=-k_{t} \delta_{i j}^{t}-2 \sqrt{\frac{5}{6}} \psi \sqrt{k_{t} m} v_{i j}^{t}
$$

where $E^{*}$ is the equivalent Young's modulus of two colliding particles, $R^{*}$ is the equivalent contact radius, $\delta_{\mathrm{ij}}{ }^{\mathrm{n}}$ refers to the normal displacement of particles under the influence of the normal force, $m^{*}$ is the equivalent mass of particles, $v_{\mathrm{ij}} \mathrm{n}^{\mathrm{i}}$ is the normal component of relative velocity. The normal contact stiffness is then calculated as $k_{n}=2 E^{*} \sqrt{R \delta_{n}}$. Damping coefficient $\mathrm{t}$ is a function of the coefficient of restitution e and ranges from 0 (absolutely viscous) to 1 (absolutely elastic). The tangential force $F_{\mathrm{ij}}{ }^{\mathrm{t}}$ is given by the tangential displacement $\delta_{\mathrm{ij}}{ }^{\mathrm{t}}$, the relative tangential velocity vijt and the tangential stiffness $k_{t}=8 G^{*} \sqrt{R \delta_{t}}$. In EDEM, the tangential force is limited by the condition defined by Coulomb's law of friction.

The input interaction parameters for the contact model such as static friction, rolling friction, and coefficient of restitution were determined experimentally by the use of experimental apparatuses. For instance, the double pendulum method for the restitution coefficient measurement is described by Hlosta et al. (2018) [32]. All virtual particulate materials were calibrated by the use of the static angle of repose (S-AoR) and the dynamic angle of repose (D-AoR) and the discharge from the calibration model hopper. The issues of discharge calibration of the particles in the DEM are described in the study of Rozbroj et al. (2019) [33]. These input data were additionally specified more exactly. No results from the calibration simulations exceeded the deviation of $5 \%$ as compared to the real calibration experiments. Examples from the C6 material calibration are shown in Figure 4.
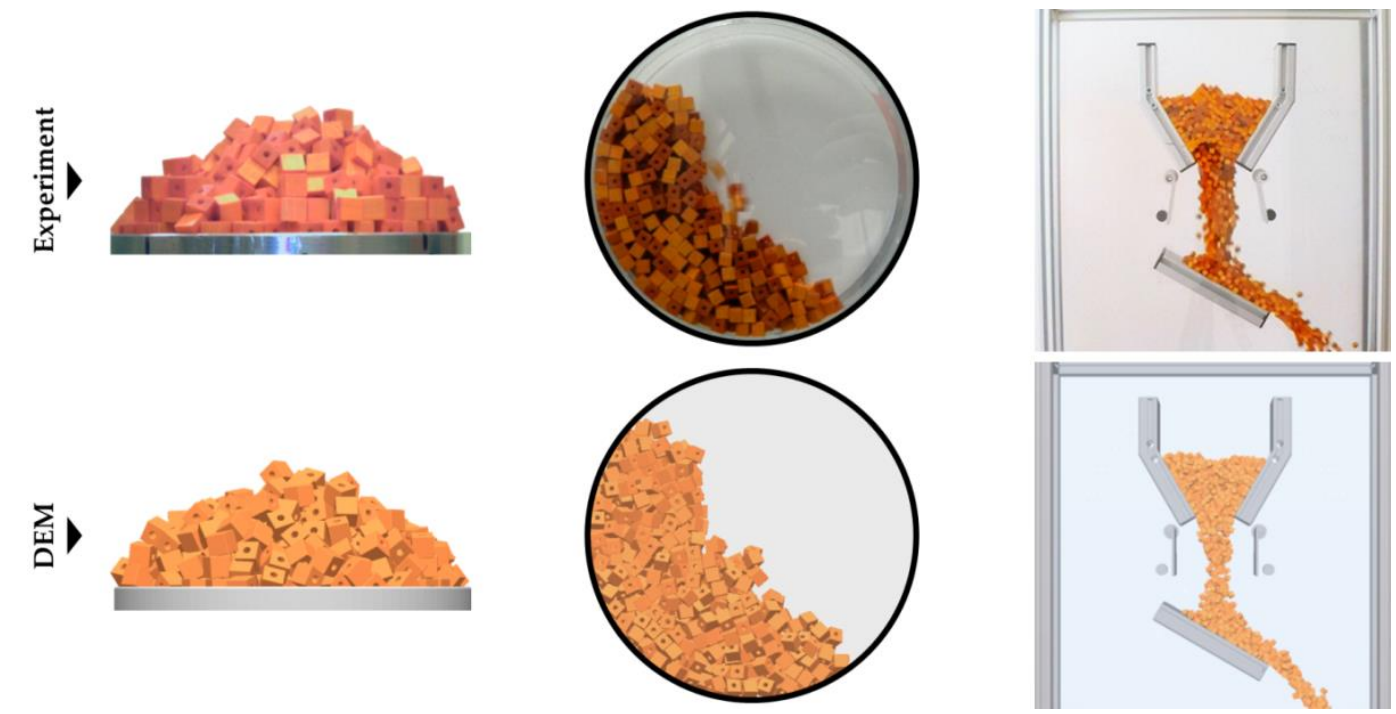

Figure 4. Calibration process of virtual material (C6 particles).

For the validation of material movement inside the drum, volume filling degrees of 30 and $60 \%$, and revolutions of 36 and $60 \mathrm{rpm}$ were chosen. In the first place, the virtual material behavior (the dynamic angle of repose, particle velocities, flow profiles, etc.,) depends on the entered interaction input parameters. Therefore, in spite of the basic calibration of the virtual material performed, it is necessary, for the use in process applications, to validate the movement of the whole complex of particles. In Figure 5, there is an example of the material movement calibration in the drum as a graphic comparison between the PIV analysis and the DEM simulation. The particle velocity visualization is provided by the use of the identical scale of colors: white (zero velocity)-green-yellow-red (velocity of $\left.0.5 \mathrm{~m} \cdot \mathrm{s}^{-1}\right)$. The green regions above the particle bed in area without the particles are caused by reflections on PMMA front wall. PIV analysis analyses the particle motion as well as the reflections motion. It is a reason why the graphical evaluation of speeds is better than numerical one. Reflections can cause a deviation in results. Operator can evaluate shape and velocities of particle bed from the graphical presentation only from the region showing particles. 


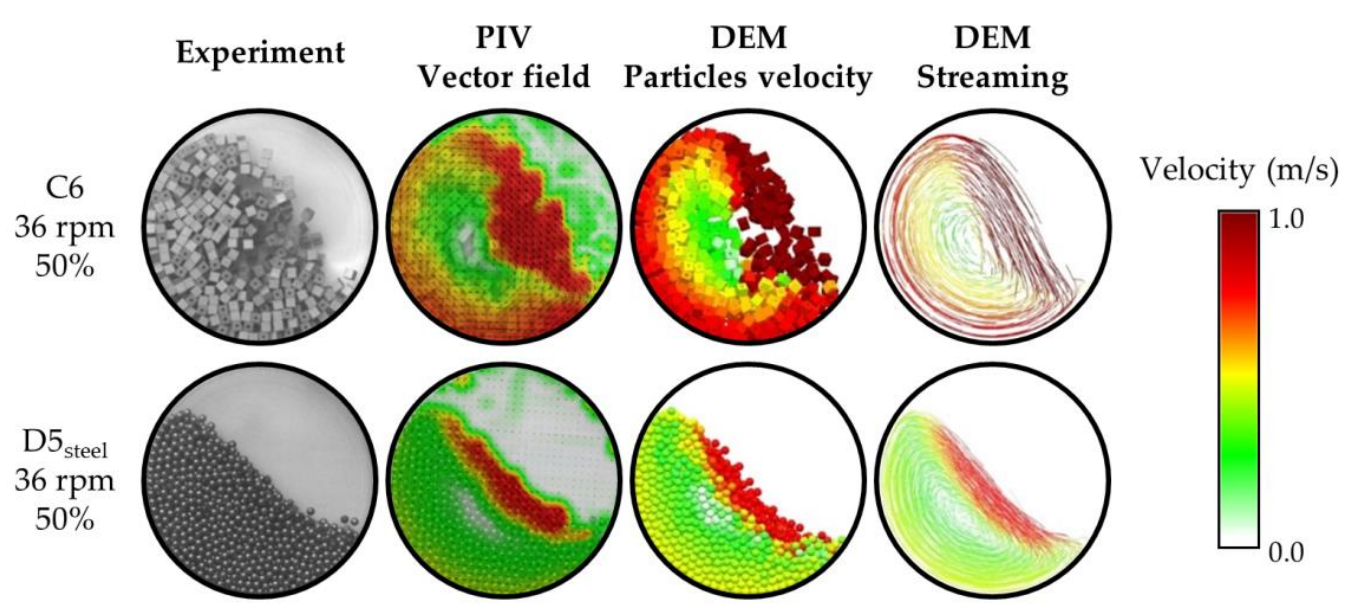

Figure 5. Validation of virtual material movement via particle image velocimetry (PIV) analysis and discrete element method (DEM) visualization.

The used numerical model was validated based on the static and dynamic angles of repose, hopper discharge times, and the PIV analysis of the material movement in the drum. The graphic evaluation between the experiments and the simulations pointed out to a good consistence. The created model was further used for movement analyses and studies of combinations of selected particles. In this way, the mixing process was graphically validated. At the identical moment, the distribution of both particulate components in the mixture was visually compared with the dynamic angle of repose at both simulation and validation experiment. An example of comparisons of selected experiments and simulation models is shown in Figure 6. Overall, 126 simulation models were validated by 28 experiments. For each particle combination 50\% drum capacity was used and 6 and $21.6 \mathrm{rpm}$, always both for vertical and horizontal filling respectively. Very good agreement was achieved between numerical models and validation experiments. This way, the particulate materials were calibrated, the mixing process validated, and it was possible to set to the numerical study.

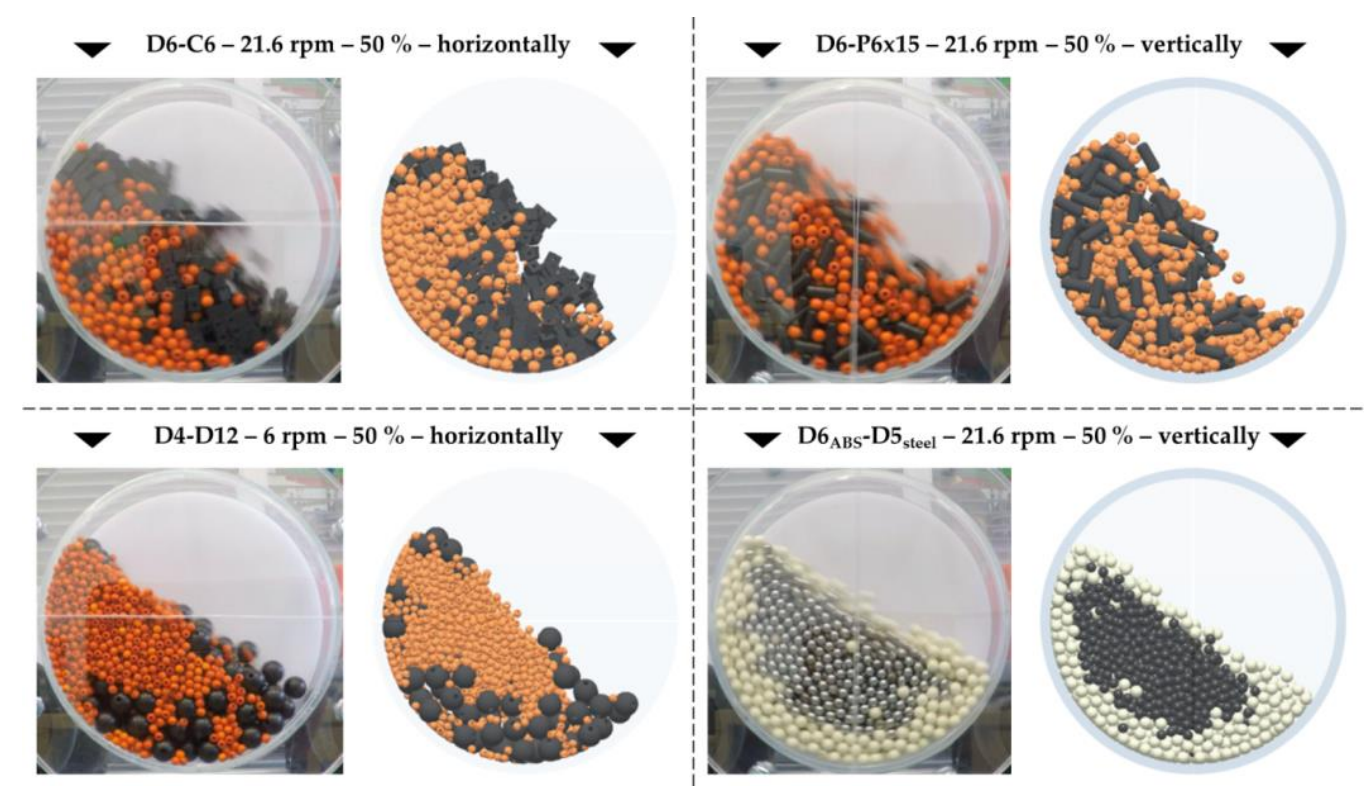

Figure 6. Validation of the binary mixture mixing process for combinations of particulates and conditions.

The results of all operating conditions were determined always based on a single simulation. This turned attention to the question of reproducibility of the results. Therefore, three simulation 
options were selected and for each of them ten simulations were created with the same numerical model, i.e., with the identical input data and operating conditions. Initial position of the particles was created randomly by "rainfalling" packing for each simulation with the same particles number. In these series of ten simulations, the homogeneity index development was observed in dependence on gradual turning of the drum. For this study, the following options were selected with regard to the tests of particle shapes, drum revolutions, and the drum filling method:

- Combination of particles D6-D6, $21.6 \mathrm{rpm}, 50 \%$ capacity filling, horizontal filling;

- Combination of particles D6-P6 $\times 15,36 \mathrm{rpm}, 50 \%$ capacity filling, vertical filling;

- Combination of particles P6 × 15-P6 × 15, $36 \mathrm{rpm}, 50 \%$ capacity filling, horizontal filling.

The reproducibility study results are shown in Figure 7. For ten repetitions of the given simulation, the homogeneity index values were obtained with the steps of 0.05 revolution of the drum (i.e., gradual turning of the drum by $18^{\circ}$ ). The data plotted into the graph create a band, the width of which defines the spread of values in the ten experiment repetitions. Out of the final values of the homogeneity index, the mean value with relative standard deviation was determined. This relative standard deviation fluctuates within the range of $R S D=\langle 0.02,0.04\rangle$, which represents the approximate error of $3 \%$. The resulting values of the homogeneity index were $0.70 \pm 0.02$ for D6-D6 particles, $0.75 \pm 0.03$ for D6-P6 $\times 15$ particles, and $0.69 \pm 0.04$ for P6 $\times 15-\mathrm{P} 6 \times 15$ particles. In both graphic outputs as well as data points, it is clear that none of the ten simulations differ in the results or its course. In all three series, a very good agreement and consistent results were achieved. The reproducibility of numerical models is therefore very good, and very credible data can be obtained even from a single simulation created.

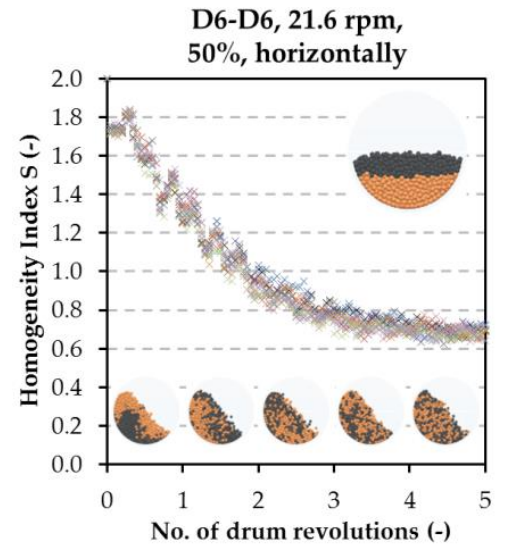

(a)

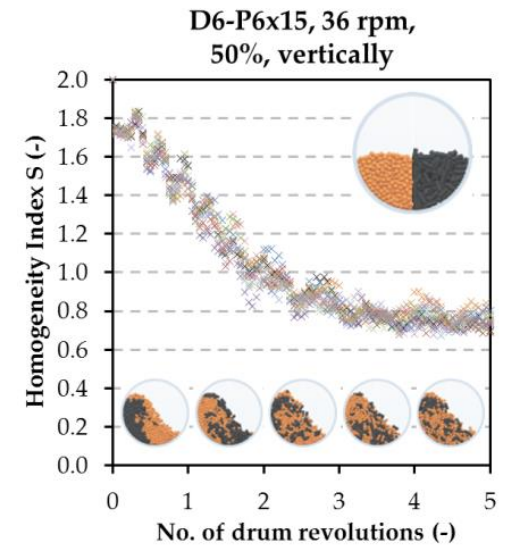

(b)

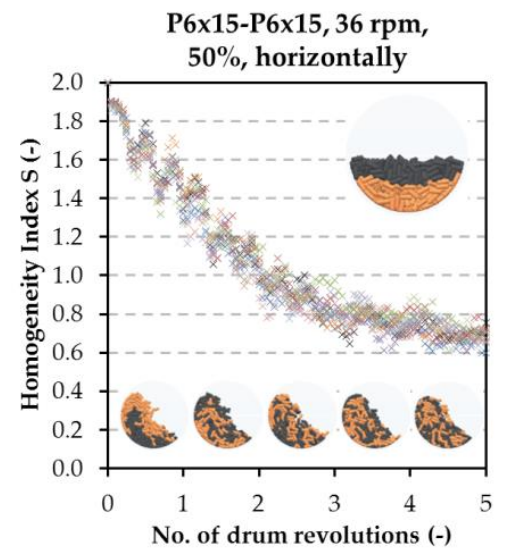

(c)

Figure 7. Reproducibility of the simulation results: (a) D6-D6, $21.6 \mathrm{rpm} 50 \%$, horizontally; (b) D6-P6 $\times$ 15, $36 \mathrm{rpm}, 50 \%$, vertically; (c) P6 × 15-P6 × 15, $36 \mathrm{rpm}, 50 \%$, horizontally.

\section{Results and Discussion}

\subsection{Influence of Particle Properties and Operating Conditions to the Mixing Process}

The first simulation series studied the mixing of D6 spherical particles. The resulting values of the homogeneity index in the mixing process in the rotary drum after 5 drum revolutions under different operating conditions for the combination of D6-D6 particles are shown in Figure 8. It is obvious from the results that the most acceptable mixing degree at 50\% of the drum filling capacity was achieved, regardless of the filling method and drum rotational speed. In this case, the drum filling capacity influences the homogeneity index to a greater degree than the drum filling method. If the drum is overfilled, the mixing degree of the mixture drops along with an increase in drum rotational speed. A good mixing quality ( $S=0.79$-see Figure 8 ) was achieved at horizontal $25 \%$ filling capacity, where diffuse mixing is applied in the sliding motion at low revolutions. In some cases of low $(25 \%$ 
horizontally) or high (75\% vertically) filling level it can be reached a high quality mixing process. The best and the most stable results were achieved at 50\% filling level. Homogeneity index slightly decreased with increasing drum rotation speed for this filling level. The horizontal filling is much better for mixing of a small amount of material at low speeds as well as for higher drum speeds.

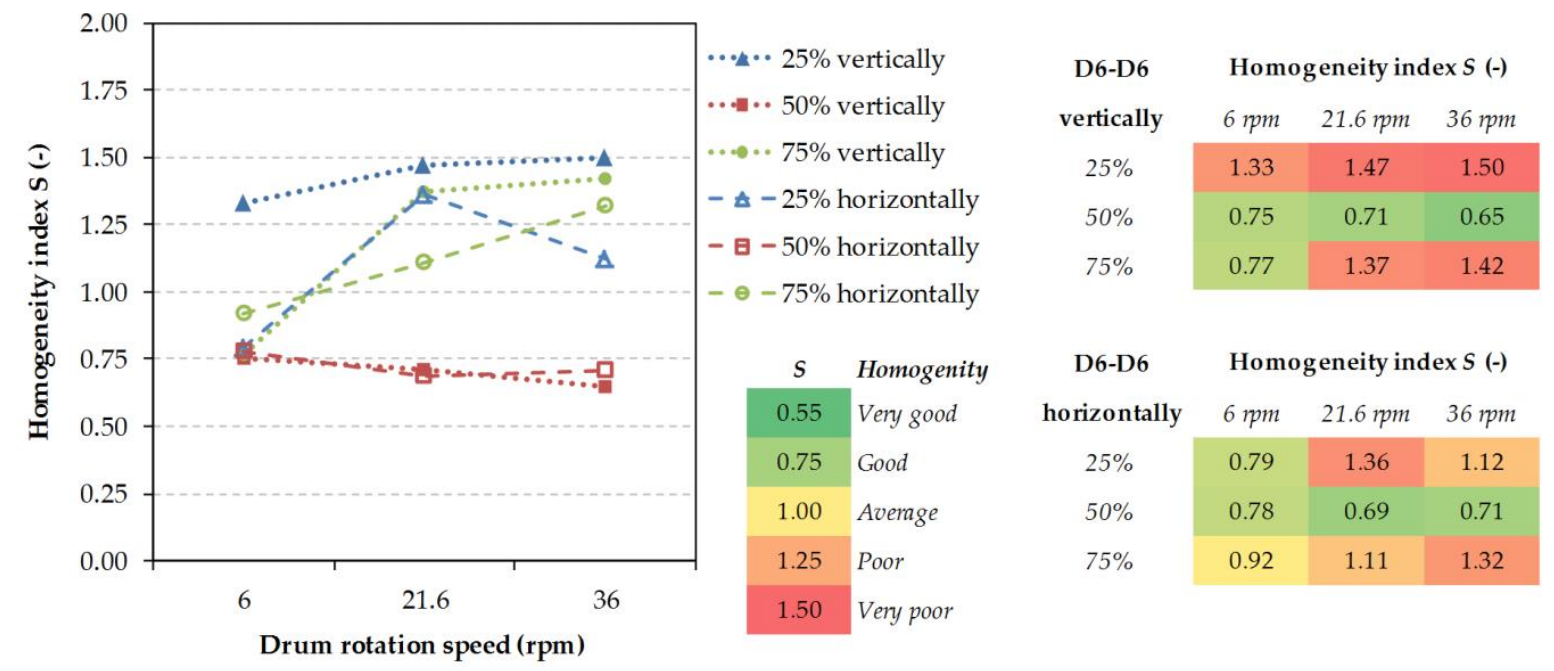

Figure 8. Resulting homogeneity indices for different operating conditions (D6-D6 particles).

In the second simulation series, the influence of the shape of particulate materials was studied. In this case, D6 spherical particles and C6 sharp-edged (cubic) particles were combined. The resulting homogeneity indices are shown in Figure 9 . The best results were achieved at $50 \%$ drum filling capacity and/or low drum rotational speed regardless of the filling method. At $25 \%$ of the drum filling capacity, the mixing degree of the mixture drops along with an increase in drum rotational speed. At $75 \%$ of the drum filling capacity, the mixing quality was the worst using the rolling movement in the drum. The most stable results were obtained at low drum rotational speed.

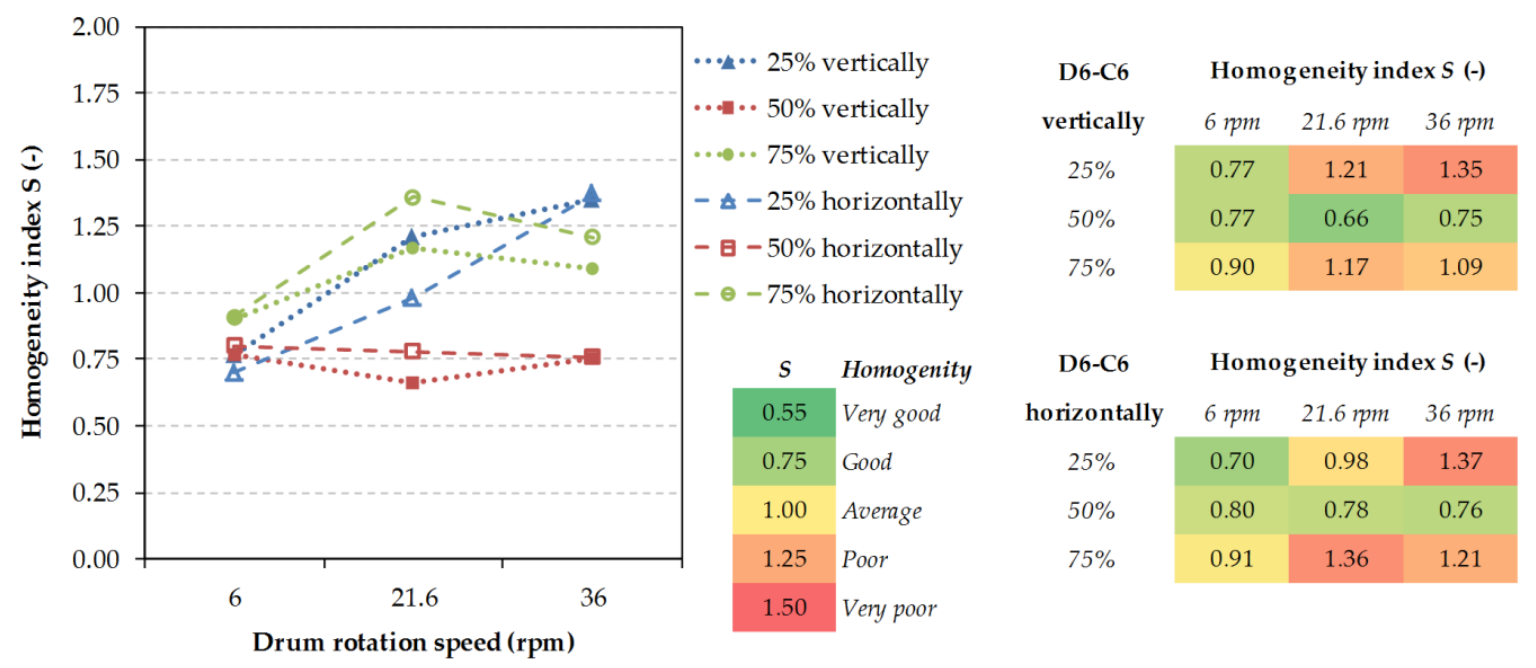

Figure 9. Resulting homogeneity indices for different operating conditions (D6-C6 particles).

In the third simulation series, the influence of the shape of particulate materials was studied. In this case, D6 spherical particles and P6 $\times 15$ cylindrical particles were combined (see Figure 10). The results showed that changing the operating parameters does not significantly influence the resulting homogeneity index for this particle shape combination. In most cases, similar results were recorded, except for simulations with overfilled drums (excluding two higher rotational speeds). In this set of 
simulations, the homogeneity index fell under the value of 1 only at $6 \mathrm{rpm}$. At higher revolutions, the mixing quality was unsatisfactory. In other cases, satisfactory mixing quality was achieved, where at lower degrees of the drum filling capacity the homogeneity index dropped more quickly. This combination of spherical and cylindrical particles showed stable results with good homogeneity indices $(S=0.75)$ after five revolutions of the drum with rotational speed of $6 \mathrm{rpm}$.

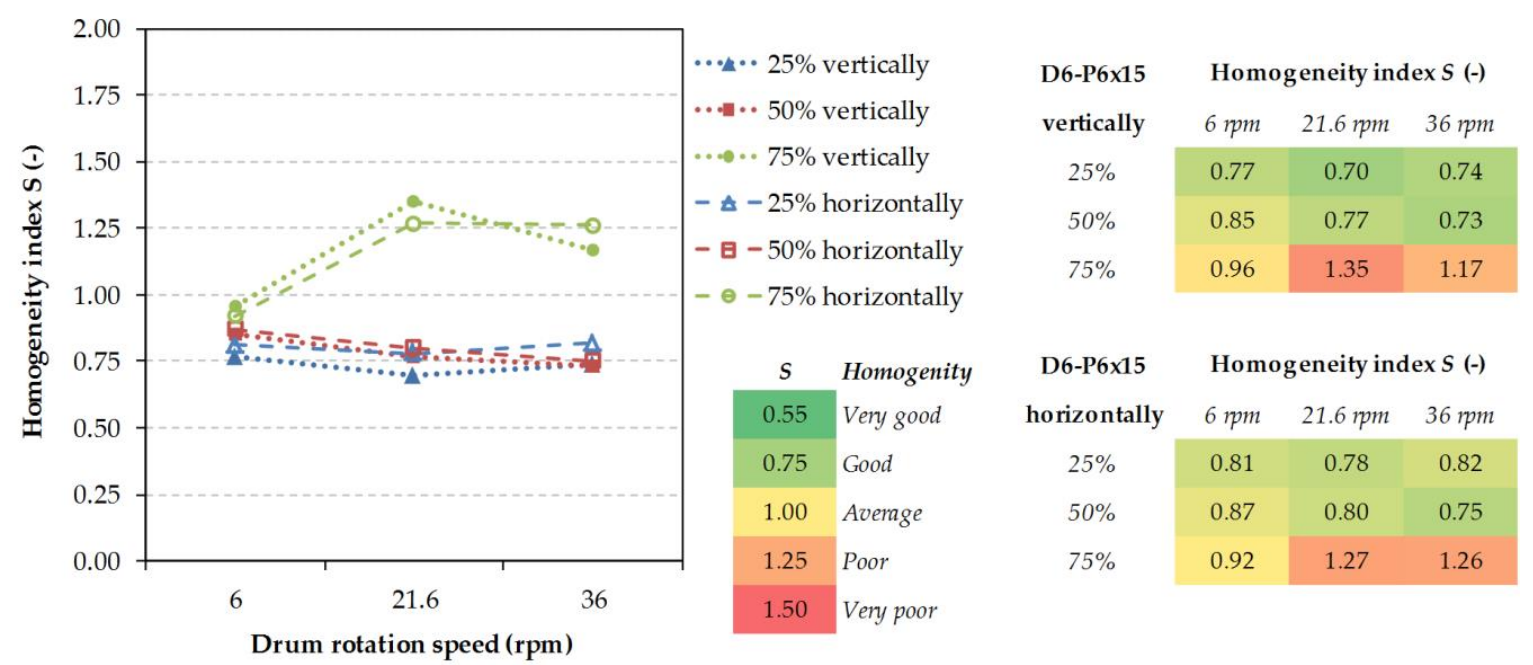

Figure 10. Resulting homogeneity indices for different operating conditions (D6-P6 $\times 15$ particles).

In the fourth simulation series, C6 sharp-edged (cubic) particles were mixed (see Figure 4). This mixture is prone to form planar contacts that prevents the rotation of particles. The resulting homogeneity indices are shown in Figure 11. Good results were achieved at $50 \%$ of the drum filling capacity, regardless of the filling method and drum rotational speed. The sharp-edged particles need a sufficient space for their loosening and mixing. The worst results were obtained at the setting of $36 \mathrm{rpm}$, $75 \%$ of the drum filling capacity, and the horizontal filling method. Acceptable results can be achieved using the horizontal filling method, however at the expense of lower revolutions and significantly extended periods of mixing. Because of the planar contacts and limited movability, surprisingly low homogeneity indices were achieved $(S=0.6)$ with some operating conditions.

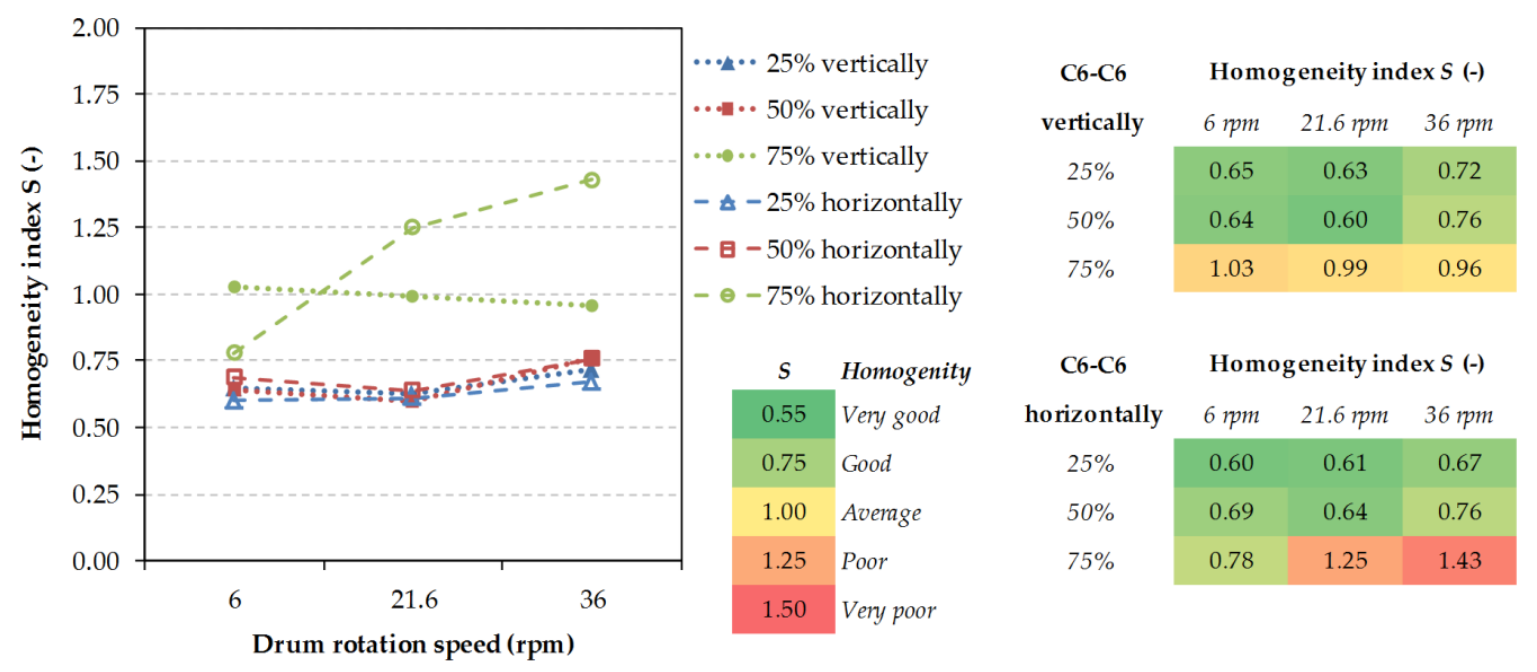

Figure 11. Resulting homogeneity indices for different operating conditions (C6-C6 particles).

The resulting homogeneity indices for the fifth simulation series are shown in Figure 12. Here, the resulting mixture consisted of cylindrical particles $\mathrm{P} 6 \times 15$. The most efficient operating mode seems to be $50 \%$ of the drum filling capacity (vertical) at low or high drum rotational speed. The mixing at low 
and middle volumes of material does not depend on the drum rotational speed, while in the overfilled drum it is more suitable to proceed well at low revolutions. Under previous simulation conditions, the low filling of the drum capacity was not very efficient because of a small amount of particles, however in the combination of $\mathrm{P} 6 \times 15-\mathrm{P} 6 \times 15$ particles the best results were achieved at $25 \%$ of the drum filling capacity. It can be assumed that the same results could be achieved also for the drum filling at $50 \%$, however at the cost of longer mixing. In such a case, a certain inaccuracy can be expected because of the smaller number of particles in the drum. The P $6 \times 15$ particle has approximately four times larger volume than the D6 spherical particle. Therefore, the number of particles needed to fill the given drum is four times lower, which reflects in the number of contacts for the homogeneity index evaluation.
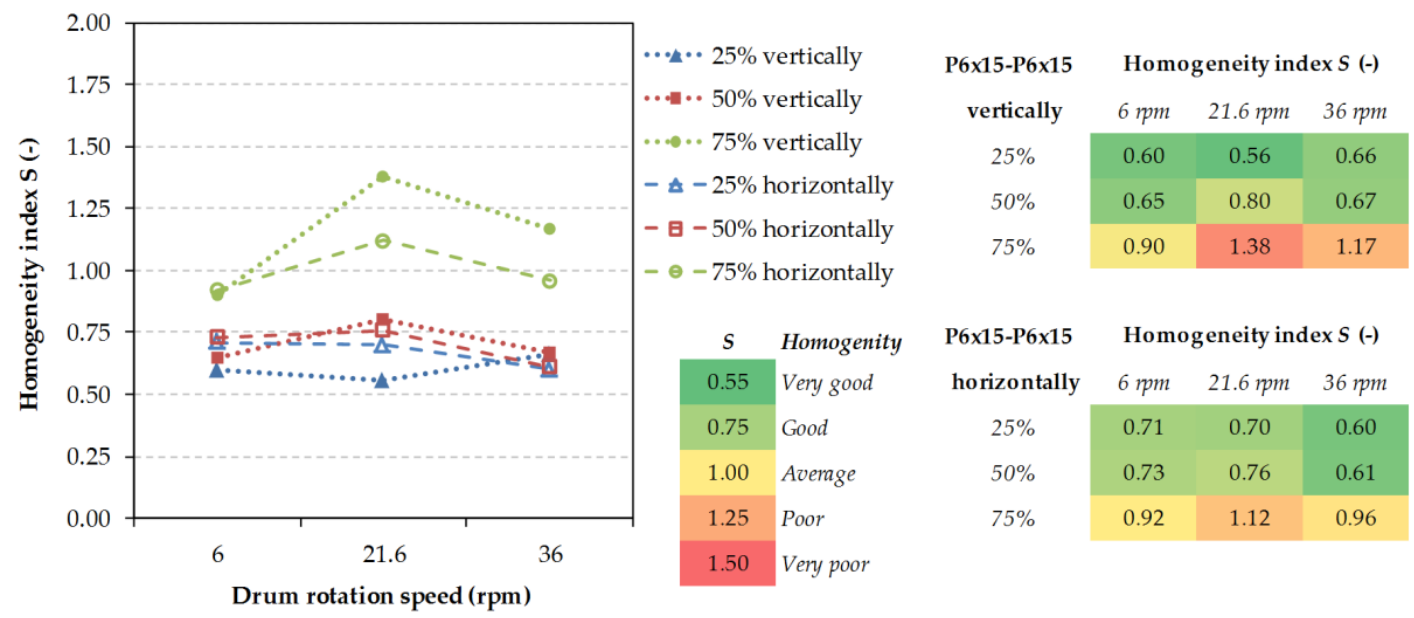

Figure 12. Resulting homogeneity indices for different operating conditions (P6 $\times 15-\mathrm{P} 6 \times 15$ particles).

In the sixth simulation series, mixing processes of particulate materials formed by spherical grains of different size, i.e., $4 \mathrm{~mm}$ and $12 \mathrm{~mm}$ were studied. The resulting homogeneity indices for the combination of D4-D12 particles (see Figure 2) are shown in Figure 13. Here, almost no differences were observed among the drum filling methods. In all cases, low revolutions demonstrated to be undesirable with the sliding mode of material movements in the drum as well as the volumetric filling at $75 \%$. At all operating settings, the D4 particles tended to aggregate in the center of the sample volume, while the larger D12 particles remained on the surface. Therefore, drum mixers seem to be unsuitable for mixing materials with a wide range of distribution of particles because the diffusion mechanism of mixing applies in the rotary drums as well as the diffusion segregation of particles. Only average homogeneity index was achieved in all cases.

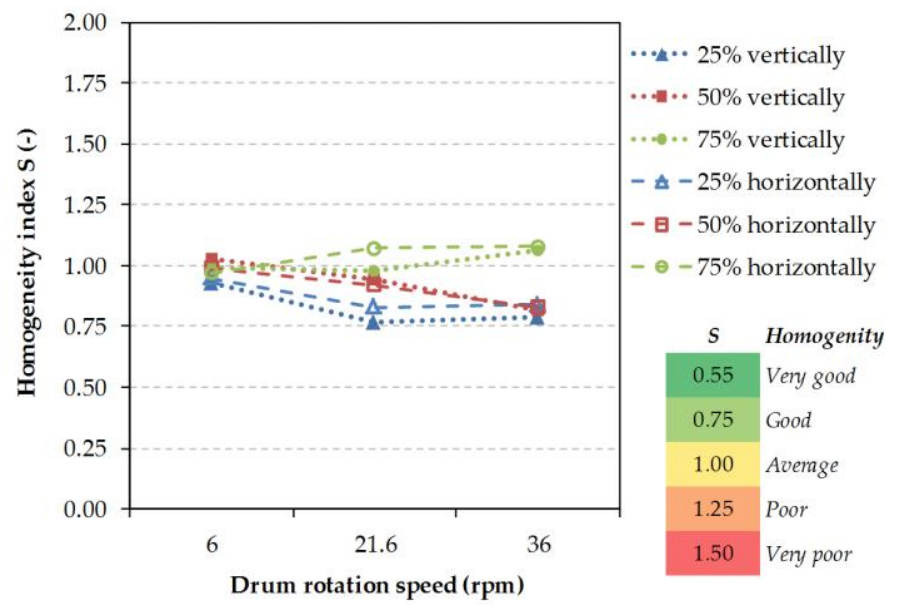

\begin{tabular}{|c|c|c|c|}
\hline D4-D12 & \multicolumn{3}{|c|}{ Homogeneity index $S(-)$} \\
\hline vertically & $6 r p m$ & $21.6 \mathrm{rpm}$ & $36 \mathrm{rpm}$ \\
\hline $25 \%$ & 0.93 & 0.77 & 0.79 \\
\hline $50 \%$ & 1.03 & 0.94 & 0.82 \\
\hline $75 \%$ & 0.99 & 0.98 & 1.06 \\
\hline D4-D12 & \multicolumn{3}{|c|}{ Homogeneity index $S(-)$} \\
\hline horizontally & $6 r \mathrm{pm}$ & $21.6 \mathrm{rpm}$ & $36 \mathrm{rm}$ \\
\hline $25 \%$ & 0.95 & 0.83 & 0.84 \\
\hline $50 \%$ & 0.99 & 0.92 & 0.83 \\
\hline $75 \%$ & 0.97 & 1.07 & 1.08 \\
\hline
\end{tabular}

Figure 13. Resulting homogeneity indices for different operating conditions (D4-D12 particles). 
In the seventh simulation series, mixing processes of particulate materials formed by spherical grains of various densities/weights in drum mixers were studied. The resulting homogeneity indices for the combination of particles $\mathrm{D} 6_{\mathrm{ABS}}-\mathrm{D} 5_{\text {steel }}$ are shown in Figure 14. The practical experiments and virtual simulations demonstrated that the drum mixers are completely unsuitable to mix particles of various densities/weights. Immediate segregation takes place in the drum, where heavier metal particles remain in the middle of the mixed sample, while they are surrounded by lighter plastic particles (see Figure 6). Likewise, with other models, only the first five revolutions of the drum were presented, however even after a longer period, no complete mixing takes place. The best results were achieved with a low filling of the drum and high revolutions, but even these results with the lowest homogeneity indices cannot be considered acceptable. The given plastic and steel particles are immiscible in standard rotary drums.

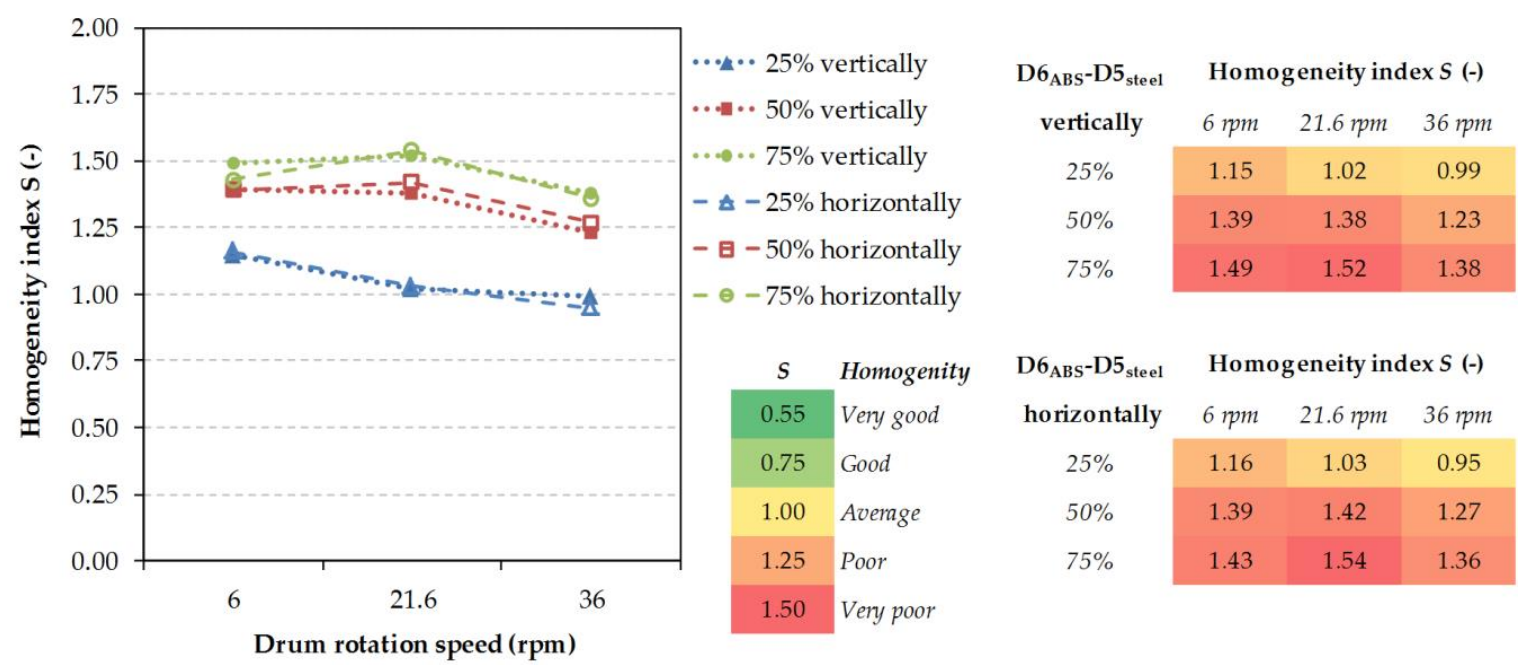

Figure 14. Resulting homogeneity indices for different operating conditions (D6 ${ }_{\mathrm{ABS}}-\mathrm{D} 5_{\text {steel }}$ particles).

\subsection{Influence of the Drum Size to the Mixing Process}

Another factor that influences the mixing processes is the size of mixing vessels, i.e., the vessel/particle size (drum/particle diameter ratio). Literary references claim that the minimum recommended drum diameter is in the range of 10 to 15 multiple of the particle size in terms of the material movements in the drum $[14,34,35]$. Qi et al. (2015) claim that in order to obtain relevant velocity fields, the suitable vessel/particle size ratio equals 50 [36]. The aim of this section is to find to what degree the homogenization process is influenced by the particle size/vessel size ratio. Therefore, three simulations were carried out for different particles and operating conditions, where three different vessel/particle size ratios were tested within the range from 10 to 70 . These conditions were chosen with regard to particle sizes and therefore combinations of D6-D6, or D6-P6 $\times 15$, and P6 $\times 15-\mathrm{P} 6 \times 15$ were selected. The operating conditions were always $36 \mathrm{rpm}$, at $50 \%$ of the drum filling and the vertical filling method. Again, the results and the homogeneity index were monitored (Figure 15) during five drum revolutions $\left(1800^{\circ}\right)$. The graphic comparison of the course of mixing in the rotary drums of the size of 420 and $140 \mathrm{~mm}$ and within the range of revolutions $0-5$ is shown in Figure 16. The results point to a slower decline in the homogeneity index for the drums of higher diameters. However, after five revolutions of the drum all three curves are interspersed at the same degree of the homogeneity index, which means that after five revolutions of the drum already, the drum size does not influence the mixing process (Figure 15). Therefore, the drum diameter of $140 \mathrm{~mm}$, i.e., the ratio of 23, used in the previous sections can be considered sufficient. The achieved result complies with the relevant literary references. It is presented that by the chosen diameter and drum revolutions, it is possible to achieve the relevant value of the homogeneity index, which would drop along with the time of the mixing process only very slowly. 


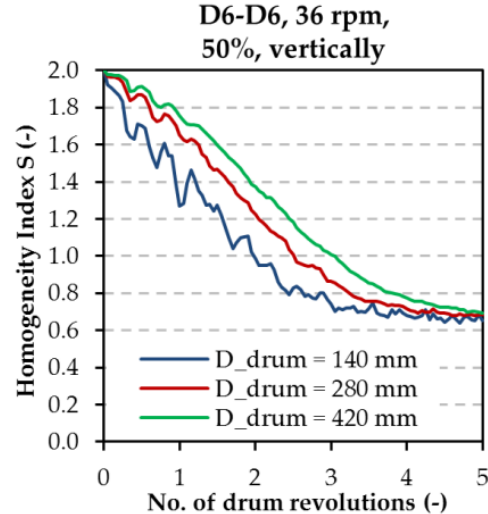

(a)

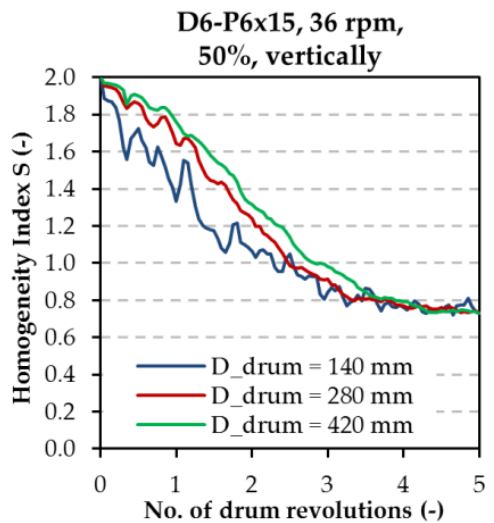

(b)

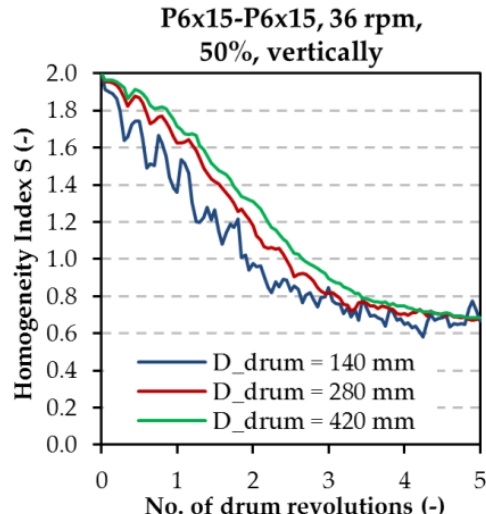

(c)

Figure 15. Homogeneity index curves for selected combinations of particles and drum sizes: (a) D6-D6, 36 rpm 50\%, vertically; (b) D6-P6 × 15, 36 rpm, 50\%, vertically; (c) P6 × 15-P6 × 15, 36 rpm, $50 \%$, vertically.

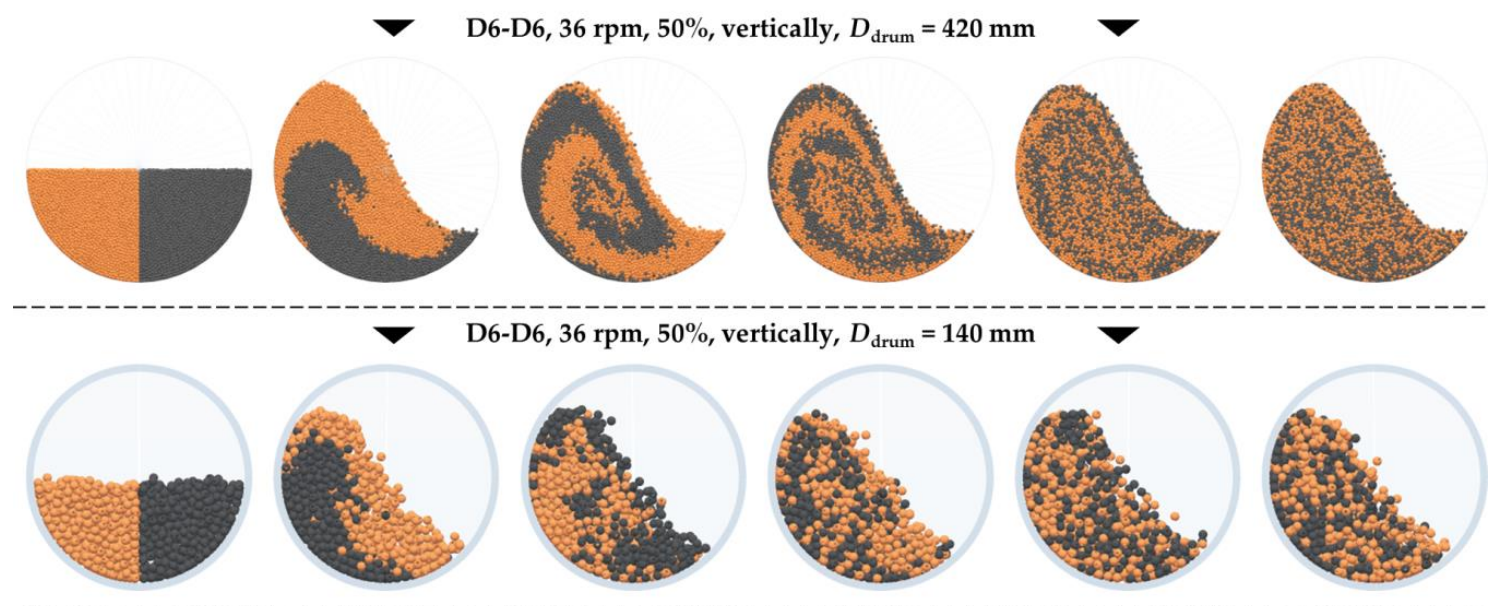

P6x15-P6x15, 36 rpm, $50 \%$, vertically $D_{\text {drum }}=420 \mathrm{~mm}$

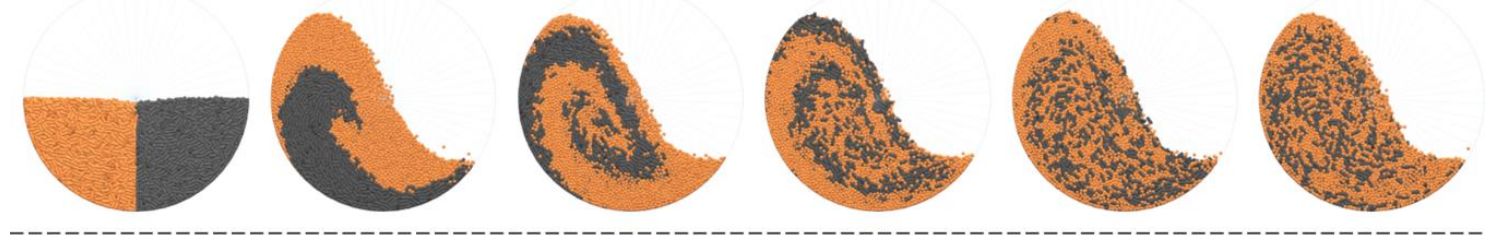

P6x15-P6x15, 36 rpm, $50 \%$, vertically $D_{\text {drum }}=140 \mathrm{~mm}$
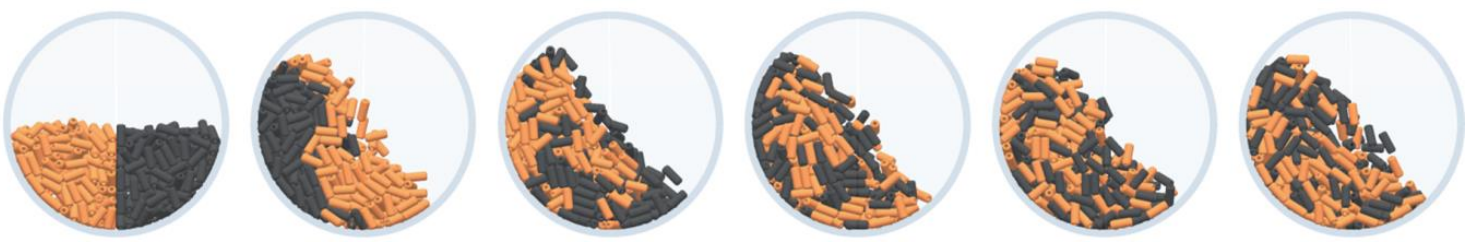

Figure 16. A graphic comparison of mixing in drums of different diameters after 0 to 5 revolutions.

\subsection{Optimal Degree of Drum Filling}

The quantities of material inside the rotary mixer significantly influences the mixing process and its efficiency [4,17]. Therefore, another series of simulations was conducted to express the dependence of mixing efficiency on the drum filling degree in a wider spectrum of the drum volumetric filling from 10 to $80 \%$. Again three mixtures of spherical D6 particles, D6-C6 spherical and sharp-edged particles, 
and C6 sharp-edged particles were chosen for this partial study. These combinations were chosen to observe the movement and rotation limitations because of the planar contacts among the sharp-edged particles. In addition, different drum diameters and drum rotational speed were considered. Complete parameters of the conditions to study the drum volumetric filling (10 to $80 \%$ ) and in $10 \%$ intervals are as follows:

- Combination of particles D6-D6, $36 \mathrm{rpm}$, horizontal filling, drum D = $280 \mathrm{~mm}$;

- Combination of particles C6 × 6-C6 × 6, 21.6 rpm, horizontal filling, drum D = $140 \mathrm{~mm}$;

- Combination of particles D6-C6 ×6,36 rpm, horizontal filling, drum D = $140 \mathrm{~mm}$.

The study results of the influence of drum filling degree on the resulting homogeneity indices are shown in Figure 17. A graphic comparison of the influence of drum filling degree in the range from 10 to $80 \%$ is shown in Figure 18 for all tested combinations of particulate materials. The curve trends (Figure 17) imply that the most efficient mixing occurs at the drum filling degree of $40-50 \%$ for spherical particles and $30-40 \%$ for sharp-edged particles and their mixtures. Apparently, the above-mentioned influence of the planar contacts among the sharp-edged particles took the effect. Therefore, in the design of a mixing process it is important to consider the shape of the particles and their possible movements in the space. The homogeneity index of the majority of cases tend to decrease because of either extremely low or, extremely high degree of drum filling. In general, it can be claimed that the drum mixers can be operated within the range from 30 to $50 \%$ of filling depending on the application, drum mixer type, and properties of particulate substances, which is fully corroborated by the visual evaluation shown in Figure 18.

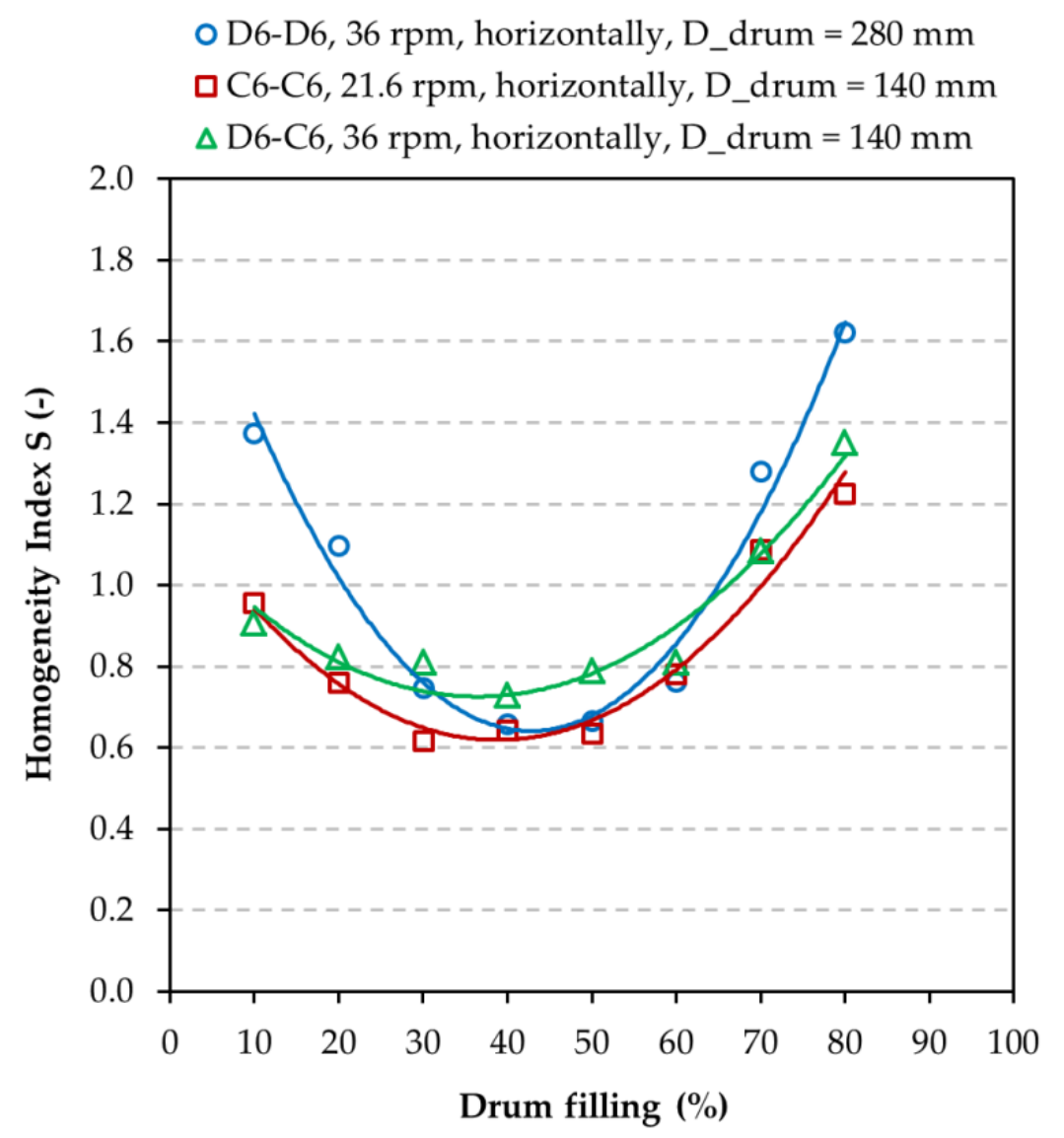

Figure 17. Influence of the degree of drum filling to the resulting homogeneity index of the mixture. 


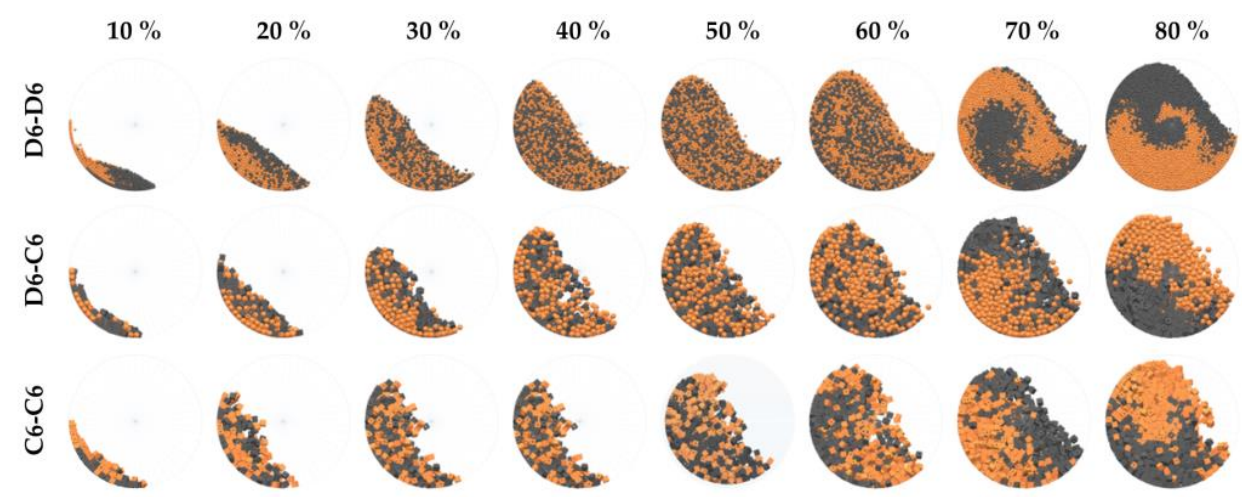

Figure 18. Influence of the degree of drum filling to the resulting homogeneity index after five revolutions.

\section{Conclusions}

The objective of this study was to compare and evaluate homogenization of seven selected particulate materials in drum mixers. The influence of particle size, shape, and density as well as volumetric filling of the drum, drum rotational speed, and drum filling pattern on the mixing process were examined. First, 126 operating conditions were created combining the materials. Simulation models were validated using 28 experiments. The homogeneity index $S$ was defined, which was consequently used to evaluate the resulting mixture homogeneity. The applicability of the discrete element method for the given drum mixer was tested. The experiments focusing on the repeatability of simulations showed the maximum relative standard deviation of the homogeneity index at $0.6 \%$ in ten identical simulations. Seven experimental and simulation series were created for the homogenization of selected particle mixtures. Each series were tested under 18 conditions for sliding ( $6 \mathrm{rpm})$, rolling (21.6 rpm), and cascade (36 rpm) material movements in the drum, for volumetric filling-up of the drum at 25,50 , and $75 \%$, and this for the simultaneous (vertical) or gradual (horizontal) method of filling.

Each subsection contains its own conclusions from concrete measurement series, however it can be claimed that the least satisfactory values of the homogeneity index were achieved in mixing particles of various densities/weights. The segregation on a greater scale revealed itself with these than with the particles of various shapes and sizes. In mixing of $4 \mathrm{~mm}$ particles with $12 \mathrm{~mm}$ particles only mediocre results were achieved at all operating conditions because of the percolation segregation, where the smaller particles fall through the larger ones. The combination of the $\mathrm{D}_{\mathrm{ABS}}-\mathrm{D} 5_{\text {steel }}$ particulate material of various densities/weights showed the highest values of the homogeneity index, even up to $S=$ 1.54. The mixture containing such a combination of particles is immiscible in an ordinary rotary drum. The lowest homogeneity indices were achieved for the mixture formed with the P $6 \times 15$ cylindrical particles. In this combination, the dominating factor was the drum filling-up degree.

In the most cases of $75 \%$ filling level non-linearity was observed for different parametric conditions. There is a "homogeneity index minimum" which is always reached at $6 \mathrm{rpm}$ (rolling material movement inside the drum). With increasing rotation speed, the likely segregation mechanisms cause a decrease in the homogeneity index. In higher speeds, the segregation process creates a reverse process to homogenization. In the case of the same sized particles (D6-D6; D6-C6; D6-P6 × 15; C6-C6) 50\% filling level seems to be the best setup for the lowest homogeneity index independent of loading pattern. In the case of different sizes and weights/density (D4-D12; D6ABS-D5steel) 25\% filling level is better than $50 \%$ filling during homogenization process. The special behavior showed "pellet-like" particles P6 $\times 15$ (D6-P6 $\times 15 ;$ P6 $\times 15-\mathrm{P} 6 \times 15)$ which are difficult to mix because of their shape. The long shape of particles makes it difficult to change particle position in particles bed volume. Because of this, a lower filling level is needed. 
In the study dealing with the influence of the drum/particle ratio very consistent and stable results were obtained. The course of the homogeneity index in $140 \mathrm{~mm}, 280 \mathrm{~mm}$, and $420 \mathrm{~mm}$ drums was very similar and after five revolutions of the drum, identical values of the homogeneity index were achieved for all the drum diameters. These experiments corroborated that the chosen drum diameter $(140 \mathrm{~mm})$ for the main experimental part of this study was sufficient and that, on the contrary, also the problems on the industrial scale can be solved assuming the simplification in the form of using larger virtual particles against the real ones. The simulation series focused on the influence of the volumetric drum filling-up on the homogenization process and illustrated the fact that the optimum degree of the drum filling is at $40-50 \%$ for the spherical particles and $30-40 \%$ for the sharp-edged particles. It can be claimed that the designed DEM model provides a possibility of detailed analyses of the mixing process in a rotary drum. Using DEM simulations and assuming an exact calibration of the model, it is possible to predict changes in the mixing process not only because of material properties (shape, size, density), but also because of process/operating conditions, such as the filling-up degree, mixing frequency, or filling method for various types of homogenizers in the industrial practice.

Author Contributions: Conceptualization, J.H. and D.Ž. Formal analysis, J.H. Funding acquisition, J.N. and J.Z. Investigation, J.H., L.J., and J.R. Methodology, J.H., L.J., and J.R. Project administration, J.N. Supervision, J.N. and J.Z. Validation, J.H., L.J., and J.R. Visualization, J.H. Writing-original draft, J.H. Writing-review and editing, L.J., J.R., and D.Ž. All authors have read and agreed to the published version of the manuscript.

Funding: This paper was conducted within the framework of the project CZ.1.05/2.1.00/19.0389: Research Infrastructure Development of the CENET and within the framework of the project SP2020/19: Research in the field of effects of abrasive bulk solids on process equipment. The paper has been done in connection with project Innovative and additive manufacturing technology-new technological solutions for 3D printing of metals and composite materials, reg. no. CZ.02.1.01/0.0/0.0/17_049/0008407 financed by Structural Founds of Europe Union.

Conflicts of Interest: The authors declare no conflict of interest.

\section{Nomenclature}

$\begin{array}{lll}\text { Symbols Used } & & \\ C & \text { number of contacts } & (-) \\ D & \text { diameter } & (\mathrm{m}) \\ E & \text { Young's modulus } & (\mathrm{Pa}) \\ e & \text { coefficient of restitution } & (-) \\ F & \text { force } & (\mathrm{N}) \\ G & \text { shear modulus } & (\mathrm{Pa}) \\ i & \text { gear ratio } & (-) \\ k & \text { contact stiffness } \\ L & \text { length } & \left(\mathrm{N} \cdot \mathrm{m}^{-1}\right) \\ m & \text { mass } & (\mathrm{m}) \\ n & \text { rotation speed } & (\mathrm{kg}) \\ R & \text { particle radius } & \left(\mathrm{min}{ }^{-1}\right) \\ S & \text { homogeneity index } & (\mathrm{m}) \\ t & \text { time } & (-) \\ v & \text { velocity } & (\mathrm{s}) \\ \text { Greek Letters } & & \left(\mathrm{m} \cdot \mathrm{s}^{-1}\right) \\ \delta & \text { displacement } & \\ \psi & \text { damping coefficient } & (\mathrm{m}) \\ \text { Subscripts } & & (-) \\ * & \text { equivalent } & \\ \text { A } & \text { particle A } & \\ \text { B } & \text { particle B } & \\ \text { i } & \text { particle i } & \\ \mathrm{j} & \text { particle j } & \\ \mathrm{n} & \text { normal } & \\ \mathrm{t} & \text { tangential } & \end{array}$




\section{Abbreviations}

$\begin{array}{ll}\text { ABS } & \text { Acrylonitrile butadiene styrene } \\ \text { AoR } & \text { Angle of repose } \\ \text { API } & \text { Active pharmaceutical ingredient } \\ \text { DEM } & \text { Discrete element method } \\ \text { PIV } & \text { Particle image velocimetry } \\ \text { PMMA } & \text { Poly-methyl methacrylate } \\ \text { RSD } & \text { Relative standard deviation }\end{array}$

\section{References}

1. Fan, L.; Chen, Y.M.; Lai, F. Recent developments in solids mixing. Powder Technol. 1990, 61, $255-287$. [CrossRef]

2. Cho, M.; Dutta, P.; Shim, J. A non-sampling mixing index for multicomponent mixtures. Powder Technol. 2017, 319, 434-444. [CrossRef]

3. Lacey, P. Developments in the theory of particle mixing. J. Appl. Chem. 1954, 4, 257-268. [CrossRef]

4. Xiao, X.; Tan, Y.; Zhang, H.; Deng, R.; Jiang, S. Experimental and DEM studies on the particle mixing performance in rotating drums: Effect of area ratio. Powder Technol. 2017, 314, 182-194. [CrossRef]

5. Danckwerts, P.V. The definition and measurement of some characteristics of mixtures. Appl. Sci. Res. Sect. A 1952, 3, 279-296. [CrossRef]

6. Tada, E.F.R.; Grajales, M.L.; Lemos, Y.P.; Thomeo, J.C. Mixture and motion of sugar cane bagasse in a rotating drum. Powder Technol. 2017, 317, 301-309. [CrossRef]

7. Mellmann, J. The transverse motion of solids in rotating cylinders-forms of motion and transition behavior. Powder Technol. 2001, 118, 251-270. [CrossRef]

8. Liu, X.Y.; Specht, E.; Gonzalez, O.G.; Walzel, P. Analytical solution for the rolling-mode granular motion in rotary kilns. Chem. Eng. Process Intensif. 2006, 45, 515-521. [CrossRef]

9. Ding, Y.I.; Seville, J.P.K.; Forster, D.J. Solids motion in rolling mode rotating drums operated at low to medium rotational speeds. Chem. Eng. Sci. 2001, 56, 1769-1780. [CrossRef]

10. Boateng, A.A. Boundary layer modelling of granular flow in the transverse plane of a partially filled rotating cylinder. Int. J. Multiph. Flow 1998, 24, 499-521. [CrossRef]

11. Xu, Y.; Xu, C.; Zhou, Z.; Du, J.; Hu, D. 2D DEM simulation of particle mixing in rotating drum: A parametric study. Particuology 2010, 8, 141-149. [CrossRef]

12. Arntz, M.M.H.D.; Otter, W.K.D.; Briels, W.J.; Bussmann, P.J.T.; Beeftinh, H.H.; Boom, R.M. Granular mixing and segregation in a horizontal rotating drum: A simulation study on the impact of rotational speed and fill level. AICHE J. 2008, 54, 3133-3146. [CrossRef]

13. Yamamoto, M.; Ishihara, S.; Kano, J. Evaluation of particle density effect for mixing behavior in a rotating drum mixer by DEM simulation. Adv. Powder Technol. 2015, 27, 864-870. [CrossRef]

14. Höhner, D.; Wirtz, S.; Scherer, V. A study on the influence of particle shape and shape approximation on particle mechanics in a rotating drum using the discrete element method. Powder Technol. 2014, 253, $256-265$. [CrossRef]

15. Wu, W.N.; Liu, X.Z.; Zhang, R.; Hu, Z. DEM investigation of the power draw for material movement in rotary drums with axis offset. Chem. Eng. Res. Des. 2019, 114, 310-317. [CrossRef]

16. Yazdani, E.; Hashemabadi, S.H. The influence of cohesiveness on particulate bed segregation and mixing in rotating drum using DEM. Physica A 2019, 525, 788-797. [CrossRef]

17. Soni, R.K.; Mohanty, R.; Mohanty, S.; Mishra, B.K. Numerical analysis of mixing of particles in drum mixers using DEM. Adv. Powder Technol. 2016, 27, 531-540. [CrossRef]

18. Liu, X.; Hu, Z.; Wu, W.; Zhan, J.; Herz, F.; Specht, E. DEM study on the surface mixing and whole mixing of granular materials in rotary drums. Powder Technol. 2017, 315, 438-444. [CrossRef]

19. McElroy, L.; Bao, J.; Yang, R.Y.; Yu, A.B. A soft-sensor approach to flow regime detection for milling processes. Powder Technol. 2009, 188, 234-241. [CrossRef]

20. Hu, Z.; Liu, X.; Wu, W. Study of the critical angles of granular material in rotary drums aimed for fast DEM model calibration. Powder Technol. 2018, 340, 563-569. [CrossRef]

21. Ottino, J.M.; Lueptow, E.M. On mixing and demixing. Science 2008, 319, 912-913. [CrossRef] [PubMed] 
22. Bbosa, L.S.; Govender, I.; Mainza, A. Development of a novel methodology to determine mill powder draw. Int. J. Miner. Process. 2016, 149, 94-103. [CrossRef]

23. He, S.Y.; Gan, J.Q.; Pinson, D.; Zhou, Z.Y. Particle shape-induced radial segregation of Binary mixtures in a rotating drum. Powder Technol. 2019, 341, 157-166. [CrossRef]

24. Khan, Z.S.; Morris, W.S. Subdiffusive axial transport of granular materials in a long drum mixer. Phys. Rev. Lett. 2005, 94, 1-4. [CrossRef] [PubMed]

25. Beakawi Al-Hashemi, H.M.; Baghabra Al-Amoudi, O.S. A review on the angle of repose of granular materials. Powder Technol. 2018, 330, 397-417. [CrossRef]

26. Raffel, M. Particle Image Velocimetry: A Practical Guide, 2nd ed.; Springer: Berlin, Germany, 2007. [CrossRef]

27. Nikolakakis, I.; Pilpel, N. Effect of particle shape on the tensile strengths of powders. Powder Technol. 1985, 42, 279-283. [CrossRef]

28. Marigo, M. Discrete Element Method Modelling of Complex Granular Motion in Mixing Vessels: Evaluation and Validation. Ph.D. Thesis, The University of Birmingham, Birmingham, UK, 2012.

29. Yan, Z.; Wilkinson, S.K.; Stitt, E.H.; Marigo, M. Investigating mixing and segregation using discrete element modelling (DEM) in the Freeman FT4 rheometer. Int. J. Pharm. 2016, 513, 38-48. [CrossRef]

30. Stambaugh, J.; Smith, Z.; Ott, E.; Losert, W. Segregation in a monolayer of magnetic spheres. Phys. Rev. E 2004, 70, 031304. [CrossRef]

31. Tsuji, Y.; Tanaka, T.; Ishida, T. Lagrangian numerical simulation of plug flow of cohesionless particles in a horizontal pipe. Powder Technol. 1992, 71, 239-250. [CrossRef]

32. Hlosta, J.; Žurovec, D.; Rozbroj, J.; Ramírez-Gómez, Á.; Nečas, J.; Zegzulka, J. Experimental determination of particle-particle restitution coefficient via double pendulum method. Chem. Eng. Res. Des. 2018, 135, $222-233$. [CrossRef]

33. Rozbroj, J.; Zegzulka, J.; Nečas, J.; Jezerská, L. Discrete element method model optimization of cylindrical pellet size. Processes 2019, 7, 101. [CrossRef]

34. Feng, Y.T.; Han, K.; Owen, D.R.J.; Loughran, J. On upscaling of discrete element models: Similarity principles. Eng. Comput. 2009, 26, 599-609. [CrossRef]

35. Coetzee, C.J. Particle upscaling: Calibration and validation of the discrete element method. Powder Technol. 2019, 344, 487-503. [CrossRef]

36. Qi, H.; Xu, J.; Zhou, G.; Chen, F.; Ge, W.; Li, J. Numerical investigation of granular flow similarity in rotating drums. Particuology 2015, 22, 119-127. [CrossRef] 